\title{
Analytical Study of Nonlinear Fractional-Order Integrodifferential Equation: Revisit Volterra's Population Model
}

\author{
Najeeb Alam Khan, ${ }^{1}$ Amir Mahmood, ${ }^{2}$ \\ Nadeem Alam Khan, ${ }^{1}$ and Asmat Ara ${ }^{1}$ \\ ${ }^{1}$ Department of Mathematical Sciences, University of Karachi, Karachi 75270, Pakistan \\ ${ }^{2}$ Department of Mathematics, COMSATS Institute of Information Technology, Lahore, Pakistan
}

Correspondence should be addressed to Najeeb Alam Khan, njbalam@yahoo.com

Received 30 May 2012; Accepted 17 October 2012

Academic Editor: Wen Chen

Copyright $\odot 2012$ Najeeb Alam Khan et al. This is an open access article distributed under the Creative Commons Attribution License, which permits unrestricted use, distribution, and reproduction in any medium, provided the original work is properly cited.

This paper suggests two component homotopy method to solve nonlinear fractional integrodifferential equations, namely, Volterra's population model. Padé approximation was effectively used in this method to capture the essential behavior of solutions for the mathematical model of accumulated effect of toxins on a population living in a closed system. The behavior of the solutions and the effects of different values of fractional-order $\alpha$ are indicated graphically. The study outlines significant features of this method as well as sheds some light on advantages of the method over the other. The results show that this method is very efficient, convenient, and can be adapted to fit a larger class of problems.

\section{Introduction}

Ecology is the study of different species in relation to their surroundings, competition for resources within and among the species, and predator-prey [1] relations among them. At times the surroundings may be infected by metabolic actions of the crowd [2,3]. In all these situations, since time rates of changes of population sizes are concerned, it is natural that the mathematical modeling be given by differential equations or integrodifferential equations. Integrodifferential equations are usually difficult to solve especially analytically, so an effective method is required to analyze the mathematical model which provides solutions conforming to physical reality. Also, fractional-order models are more accurate than integerorder models, that is, there are more degrees of freedom in the fractional-order models. Furthermore, fractional derivatives provide an excellent instrument for the description of 
memory and hereditary properties of various materials and processes due to the existence of a "memory" term in the model. This memory term insures the history and its impact to the present and future.

Problems of this type have gained escalating importance in recent years and many interesting outcomes have been accumulated. In the living thing population, the accumulation of metabolic products may cause inconvenience to the whole population and may ultimately result in a fall of the birth rate while the death rate is increased. We assumed that the total toxic effect on birth and death rates be expressed by the following nonlinear fractional-order integrodifferential equation [4]:

$$
\frac{d^{\alpha} p(\bar{t})}{d \bar{t}^{\alpha}}=p(\bar{t})\left(a-b p(\bar{t})-c \int_{0}^{\bar{t}} p(x) d x\right), \quad p(0)=k, 0<\alpha \leq 1,
$$

where $a>0$ is the birth rate coefficient, $b>0$ is the crowding coefficient, and $c>0$ is the toxicity coefficient. The coefficient $c$ indicates the essential behavior of the population evolution before its level falls to zero in the long term, $k$ is the initial population, $p=p(\bar{t})$ denotes the population at time $\bar{t}$, and the term $p(\bar{t}) \int_{0}^{\bar{t}} p(x) d x$ represents the effect of toxin accumulation on the species. The individual death rate is proportional to this integral, and so the population death rate due to toxicity must include a factor $\alpha$. We apply the scale time and population by introducing the nondimensional variables $t=c \bar{t} / b, v=b p / a$ to obtain the following nondimensional problem:

$$
\kappa \frac{d^{\alpha} v(t)}{d t^{\alpha}}-v(t)+v^{2}(t)+v(t) \int_{0}^{t} v(x) d x=0, \quad v(0)=v_{0}, 0<\alpha \leq 1
$$

where $v(t)$ is the scaled population of identical individuals at time $t$, and $\kappa=c / a b$ is a prescribed nondimensional parameter. The only equilibrium solution of (1.2) is the trivial solution $v(t)=0$.

Several numerical and analytical methods for approximating the ordinary nonlinear Volterra's integrodifferential equation are known (see [5-12]).

In the present paper, we introduce a two component homotopy perturbation method [13-15] for solving nonlinear fractional-order integrodifferential population model. It is interesting to note that the method depends only on two components of the homotopy series. The method is an improvement of the classical homotopy perturbation method [16].

\section{Approximations of the Nonlinear Fractional-Order Integrodifferential Equation}

To obtain the solution of (1.2) by two components HPM, we construct the following homotopy:

$$
(1-\varepsilon)\left(D_{t}^{\alpha} V(t)-v_{0}(t)\right)+\varepsilon\left(D_{t}^{\alpha} V(t)-\frac{1}{\mathcal{K}}\left(V(t)-V^{2}(t)-V(t) \int_{0}^{t} V(x) d x\right)\right)=0
$$


International Journal of Differential Equations

Applying the inverse operator, $J_{t}^{\alpha}$ to both sides of (2.1), we obtain

$$
V(t)=V(0)+J_{t}^{\alpha} v_{0}(t)-\varepsilon J_{t}^{\alpha}\left(v_{0}(t)-\frac{1}{\mathcal{K}}\left(V(t)-V^{2}(t)-V(t) \int_{0}^{t} V(t) d t\right)\right)
$$

The solution of (2.2) to have the following form

$$
V(t)=V_{0}(t)+\varepsilon V_{1}(t)
$$

Substituting (2.3) in (2.2) and equating the coefficients of like powers of $\varepsilon$, we get

$$
\begin{aligned}
& V_{0}(t)=V(0)+J_{t}^{\alpha} v_{0}(t) \\
& V_{1}(t)=J_{t}^{\alpha}\left(-v_{0}(t)+\frac{1}{\mathcal{K}}\left(V_{0}(t)-V_{0}^{2}(t)-V_{0}(t) \int_{0}^{t} V_{0}(x) d x\right)\right) .
\end{aligned}
$$

Assuming $v_{0}(t)=\sum_{n=0}^{10} a_{n} t^{n}, V(0)=v(0)$ then (2.4) becomes

$$
V_{0}(t)=\frac{a_{0} t^{\alpha}}{\Gamma(\alpha+1)}+\frac{a_{1} t^{1+\alpha}}{\Gamma(\alpha+2)}+\frac{2 a_{2} t^{2+\alpha}}{\Gamma(\alpha+3)}+\frac{6 a_{3} t^{3+\alpha}}{\Gamma(\alpha+4)}+\cdots+\frac{3629800 a_{10} t^{10+\alpha}}{\Gamma(\alpha+11)}
$$

Utilizing (2.6) in (2.5), we obtain

$$
V_{1}(t)=\frac{4 a_{0} t}{5 \kappa}+\frac{4 a_{1} t^{2+\alpha}}{5 \kappa \Gamma(\alpha+3)}-\frac{2 a_{2} t^{2+\alpha}}{\Gamma(\alpha+3)}-\frac{3 a_{0} t^{2+\alpha}}{10 \kappa \Gamma(\alpha+3)}+\cdots
$$

Vanishing $V_{1}(t)$ with $\alpha=1$ and comparing the coefficients of $t, t^{2}, t^{3}, t^{4}, \ldots$, we obtain the coefficients as follows:

$$
\begin{aligned}
& t^{1}:-a_{0}+\frac{9}{100 \kappa}+\frac{4 a_{0}}{5 \kappa}=0, \\
& t^{2}:-\frac{1}{200 \kappa}-\frac{4 a_{1}}{2}=0, \\
& t^{3}:-\frac{a_{2}}{3}-\frac{a_{0}}{20 \kappa}-\frac{a_{0}^{2}}{3 \kappa}+\frac{2 a_{1}}{15 \kappa}=0, \\
& t^{4}:-\frac{a_{3}}{4}-\frac{a_{0}^{2}}{8 \kappa}-\frac{a_{1}}{60 \kappa}+\frac{a_{0} a_{1}}{4 \kappa}+\frac{a_{2}}{15 \kappa}=0,
\end{aligned}
$$


After solving the system of (2.8), we obtain the solutions of (1.2) as follows:

$$
\begin{aligned}
v(t)= & \frac{1}{10}+\frac{9 t^{\alpha}}{20(5 \kappa-4) \Gamma(\alpha+1)}-\frac{t^{\alpha+1}}{100 \kappa \Gamma(\alpha+2)}+\frac{\left(-128+455 \kappa-875 \kappa^{2}\right) t^{\alpha+2}}{1000 \kappa^{2}(5 \kappa-4)^{2} \Gamma(\alpha+3)} \\
& +\frac{\left(-1024+3200 \kappa-13325 \kappa^{2}+1000 \kappa^{3}\right) t^{\alpha+3}}{10000 \kappa^{3}(5 \kappa-4)^{2} \Gamma(\alpha+4)}+\cdots
\end{aligned}
$$

When $\alpha=1$, we recovered [5]. Our objective is to find the behavior of the solutions for different values of the parameter $\alpha$. In order to find the behavior of the solution for fractional values of $\alpha$, we use Padé approximants which have the advantage of manipulating the polynomial approximation into a rational function to gain more information about $v(t)$. It is well known that Padé approximants will converge on the entire real axis if the solution of the problem is free of singularities on the real axis. Here, we consider the following two special cases.

Case I. We inspect the case $\alpha=1 / 2$, we reproduce the approximate solution obtained in (2.9) given by the Taylor expansion of $V(t)$ at $t=0$ which gives

$$
V(t)=\frac{1}{10}+\frac{9 \sqrt{t}}{10(5 \kappa-4) \sqrt{\pi}}-\frac{t^{3 / 2}}{75 \kappa \sqrt{\pi}}+\frac{\left(-128+455 \kappa-875 \kappa^{2}\right) t^{5 / 2}}{1875 \kappa^{2}(5 \kappa-4)^{2} \sqrt{\pi}}+\cdots
$$

For simplicity, let $t^{1 / 2}=z$, then

$$
\begin{aligned}
V(z)= & \frac{1}{10}+\frac{9 z}{10(5 \kappa-4) \sqrt{\pi}}-\frac{z^{3}}{75 \kappa \sqrt{\pi}}+\frac{\left(-128+455 \kappa-875 \kappa^{2}\right) z^{5}}{1875 \kappa^{2}(5 \kappa-4)^{2} \sqrt{\pi}}+\cdots \\
& +\frac{-8\left(-68719476736+2601139568640 \kappa+\cdots+55821093750000 \kappa^{10}\right) z^{21}}{671353055419921875 \kappa^{2}(5 \kappa-4)^{6} \sqrt{\pi}} .
\end{aligned}
$$

Calculating the [10/10] Padé approximants and recalling that $z=t^{1 / 2}$, we get

$$
V_{[10 / 10]}(t)=\frac{0.1+m_{1} t^{1 / 2}+m_{2} t+m_{2} t^{3 / 2} \cdots+m_{r} t^{5}}{n_{0}+n_{1} t+n_{2} t^{2}+n_{3} t^{3} \cdots+n_{s} t^{5}}
$$

where $m_{i}$ and $n_{i}, i=0,1,2$ are constants.

Case II. Now, we observe the case $\alpha=3 / 4$, we reproduce the approximate solution obtained in (2.9) given by the Taylor expansion of $V(t)$ at $t=0$ which gives

$$
V(t)=\frac{1}{10}+\frac{9 t^{3 / 4}}{20(5 \kappa-4) \Gamma(7 / 4)}-\frac{t^{7 / 4}}{100 \kappa \Gamma(11 / 4)}+\frac{\left(-128+455 \kappa-875 \kappa^{2}\right) t^{11 / 4}}{1000 \kappa^{2}(5 \kappa-4)^{2} \Gamma(15 / 4)}+\cdots
$$




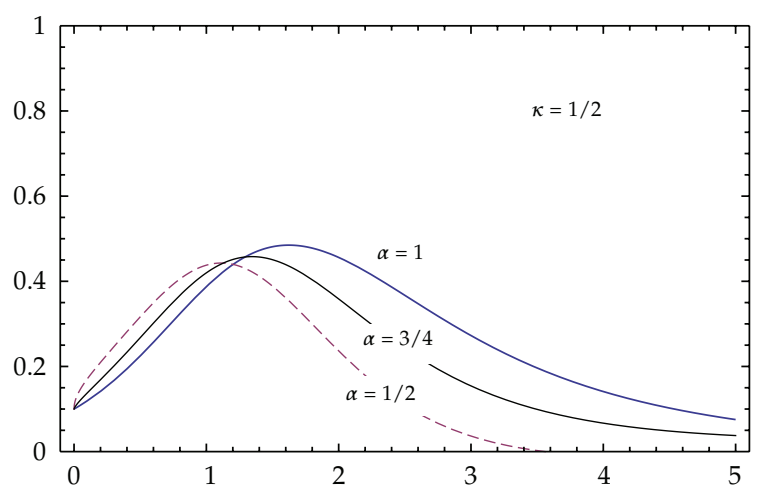

Figure 1: Approximate curves of fractional-order integrodifferential equation $t$ versus $v$.

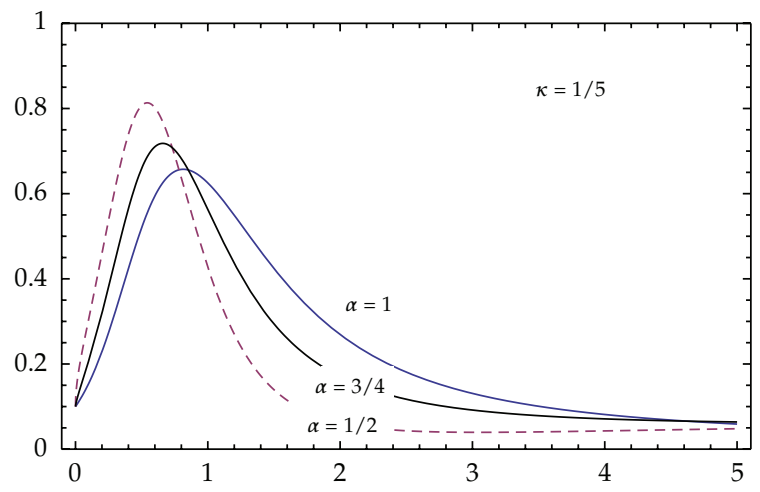

Figure 2: Approximate curves of fractional-order integrodifferential equation $t$ versus $v$.

For simplicity, let $t^{1 / 4}=z$, then

$$
\begin{aligned}
V(z)= & \frac{1}{10}+\frac{9 z^{3}}{20(5 \kappa-4) \Gamma(7 / 4)}-\frac{z^{7}}{100 \kappa \Gamma(11 / 4)}+\frac{\left(-128+455 \kappa-875 \kappa^{2}\right) z^{11}}{1000 \kappa^{2}(5 \kappa-4)^{2} \Gamma(15 / 4)}+\cdots \\
& +\frac{-8\left(-68719476736+2601139568640 \kappa+\cdots+55821093750000 \kappa^{10}\right) z^{43}}{12500000000 \kappa^{10}(5 \kappa-4)^{6} \Gamma(47 / 4)}
\end{aligned}
$$

Calculating the [21/21] Padé approximants and recalling that $z=t^{1 / 4}$, we get

$$
v_{[21 / 21]}(t)=\frac{0.1+M_{1} t^{1 / 4}+M_{2} t^{3 / 4}+M_{2} t^{5 / 4} \cdots+M_{r} t^{43 / 4}}{N_{0}+N_{1} t+N_{2} t^{2}+N_{3} t^{3} \cdots+N_{s} t^{t^{4}}} .
$$

Figure 1 shows the numerical solutions generated by the two component HPM and Padé approximation for various values of $\alpha$ and $\kappa=1 / 2$. It should be noted that solution falls slowly when value of $\alpha$ decreases. One trend that can be observed in Figures 2, 3, 4, and 5 shows the two component HPM combining with Padé approximants shows the quick rise along the logistic curve followed by the slow exponential decay later than 


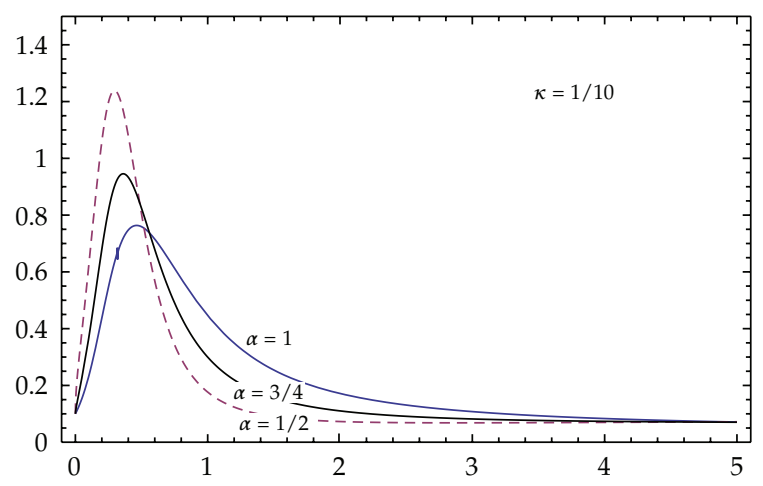

Figure 3: Approximate curves of fractional-order integrodifferential equation $t$ versus $v$.

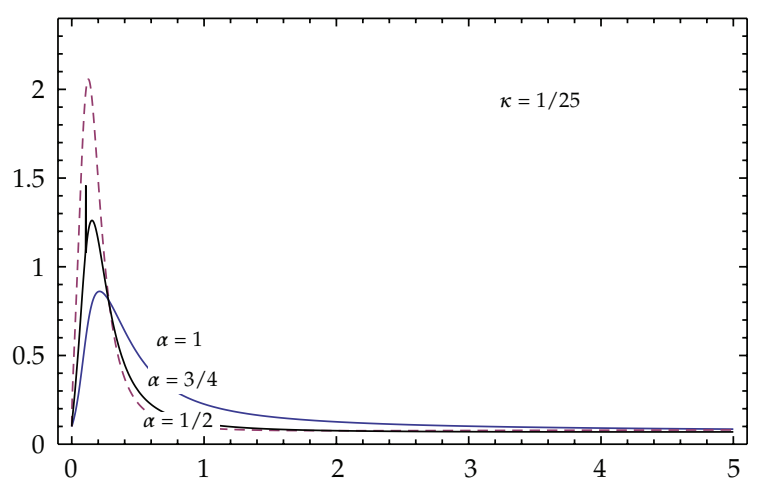

Figure 4: Approximate curves of fractional-order integrodifferential equation $t$ versus $v$.

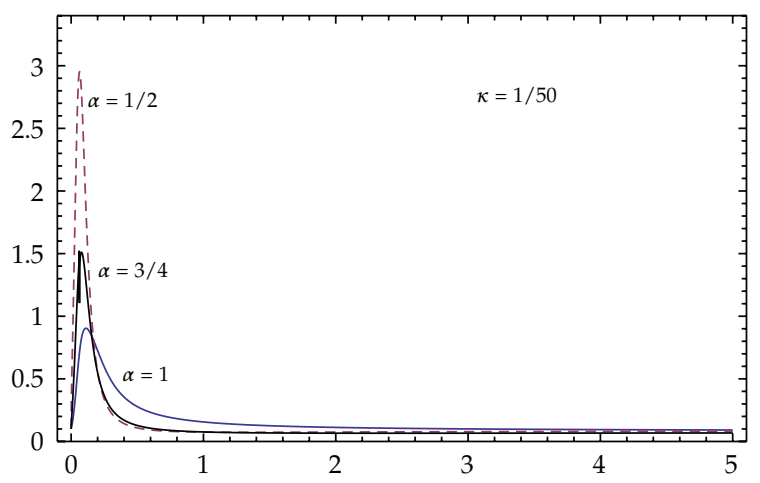

Figure 5: Approximate curves of fractional-order integrodifferential equation $t$ versus $v$.

reaching the maximum point when $\kappa$ increases, the amplitude of $v(t)$ decreases whereas the exponential decay increases, for $\kappa=1 / 50,1 / 25,1 / 10,1 / 5$. It should be noted that the solution quickly rises when value of $\alpha$ increasing. These figures give the two component HPM-Padé approximations for some values of $\alpha$. From these figures, it is shown that the maximum of $v(t)$ deceases as $\kappa$ increases. Figures $1-5$ show the impact of fractional time parameter and $\kappa$ on the behavior of population. 
Table 1: A comparison of two component HPM-Padé with the exact values of $v_{\max }$ for different fractionalorder.

\begin{tabular}{lcccc}
\hline$\kappa$ & $\begin{array}{c}\text { Exact } \\
v_{\max }\end{array}$ & $\begin{array}{c}\text { Reference [5] } \\
\alpha=1\end{array}$ & $\begin{array}{c}\text { Present } \\
\alpha=3 / 4\end{array}$ & $\begin{array}{c}\text { Present } \\
\alpha=1 / 2\end{array}$ \\
\hline 0.02 & 0.92342717 & 0.922942037 & 1.49200000 & 2.92000000 \\
0.04 & 0.87371998 & 0.873725344 & 1.24600000 & 2.01600000 \\
0.1 & 0.76974149 & 0.765113089 & 0.94150000 & 1.23200000 \\
0.2 & 0.65905038 & 0.659050432 & 0.71690000 & 0.81130000 \\
0.5 & 0.48519030 & 0.485190290 & 0.45740000 & 0.44320000 \\
\hline
\end{tabular}

Table 2: A comparison of $t_{\text {Present }}$ with the exact values and fractional values of critical $t$.

\begin{tabular}{lcccc}
\hline$\kappa$ & $\begin{array}{c}t_{\text {Exact }} \\
\alpha=1\end{array}$ & $\begin{array}{c}\text { Reference [5] } \\
\alpha=1\end{array}$ & $\begin{array}{c}t_{\text {Present }} \\
\alpha=3 / 4\end{array}$ & $\begin{array}{c}t_{\text {Present }} \\
\alpha=1 / 2\end{array}$ \\
\hline 0.02 & 0.11184543 & 0.11184543 & 0.07810000 & 0.06291000 \\
0.04 & 0.21024644 & 0.21024644 & 0.15400000 & 0.12370000 \\
0.1 & 0.46447670 & 0.46447670 & 0.35150000 & 0.29070000 \\
0.2 & 0.81685810 & 0.81685810 & 0.67050000 & 0.54900000 \\
0.5 & 1.62671100 & 1.62594670 & 1.33900000 & 1.12600000 \\
\hline
\end{tabular}

\section{Conclusions}

In this paper, two component homotopy-Padé solutions have been presented for the fractional-order integrodifferential equation. We have calculated the solutions for two different cases $\alpha=3 / 4,1 / 2$. The solutions of the population problem will be helpful in past/future predictions due to memory term. This version of HPM is independent of solving functionaldifferential equations. Also, the procedure can be applied for the system of fractional-order integrodifferential equations. The obtained outcome indicates that this method is powerful and has an important effect for solving the nonlinear fractional-order equations. Tables 1 and 2 represents the numerical comparison of ordinary and fractional order results for maximum population and critical time. Finally, the solutions of the problem for $\alpha=1$ have been reported in [5].

\section{Acknowledgment}

The corresponding authors of this paper wish to thank the reviewer for their useful comments and suggestions towards the improvement of this paper. The first author is thankful to the Dean of the faculty of Sciences, of the University of Karachi for supporting this work.

\section{References}

[1] N. A. Khan, N. U. Khan, M. Ayaz, and J. A. Siddiqui, "A numerical comparision for the fractional order Lotka-Voltrra equations," World Applied Sciences Journal, vol. 12, no. 12, pp. 2240-2246, 2011.

[2] F. M. Scudo, "Vito Volterra and theoretical ecology," Theoretical Population Biology, vol. 2, no. 1, pp. 1-23, 1971.

[3] R. D. Small, "Population growth in a closed system," SIAM Review, vol. 25, no. 1, pp. 93-95, 1983.

[4] H. Xu, "Analytical approximations for a population growth model with fractional order," Communications in Nonlinear Science and Numerical Simulation, vol. 14, no. 5, pp. 1978-1983, 2009. 
[5] N. A. Khan, A. Ara, and M. Jamil, "Approximations of the nonlinear Volterra's population model by an efficient numerical method," Mathematical Methods in the Applied Sciences, vol. 34, no. 14, pp. 1733-1738, 2011.

[6] K. Parand and M. Razzaghi, "Rational Chebyshev tau method for solving Volterra's population model," Applied Mathematics and Computation, vol. 149, no. 3, pp. 893-900, 2004.

[7] K. G. TeBeest, "Numerical and analytical solutions of Volterra's population model," SIAM Review, vol. 39, no. 3, pp. 484-493, 1997.

[8] A. M. Wazwaz, "Analytical approximations and Padé approximants for Volterra's population model," Applied Mathematics and Computation, vol. 100, no. 1, pp. 13-25, 1999.

[9] K. Parand and G. Hojjati, "Solving Volterra's population model using new second derivative multistep methods," American Journal of Applied Sciences, vol. 5, no. 8, pp. 1019-1022, 2008.

[10] K. Parand, Z. Delafkar, N. Pakniat, A. Pirkhedri, and M. K. Haji, “Collocation method using sinc and Rational Legendre functions for solving Volterra's population model," Communications in Nonlinear Science and Numerical Simulation, vol. 16, no. 4, pp. 1811-1819, 2011.

[11] H. R. Marzban, S. M. Hoseini, and M. Razzaghi, "Solution of Volterra's population model via block-pulse functions and Lagrange-interpolating polynomials," Mathematical Methods in the Applied Sciences, vol. 32, no. 2, pp. 127-134, 2009.

[12] S. T. Mohyud-Din, A. Yildirim, and Y. Gülkanat, "Analytical solution of Volterra's population model," Journal of King Saud University-Science, vol. 22, no. 4, pp. 247-250, 2010.

[13] N. A. Khan, A. Ara, and M. Jamil, "An efficient approach for solving the Riccati equation with fractional orders," Computers and Mathematics with Applications, vol. 61, no. 9, pp. 2683-2689, 2011.

[14] N. A. Khan, M. Jamil, A. Ara, and N. U. Khan, "On efficient method for system of fractional differential equations," Advances in Difference Equations, vol. 2011, Article ID 303472, 2011.

[15] N. A. Khan, M. Jamil, A. Ara, and S. Das, "Explicit solution of time-fractional batch reactor system," International Journal of Chemical Reactor Engineering, vol. 9, article A91, 2011.

[16] J. H. He, "Coupling method of a homotopy technique and a perturbation technique for non-linear problems," International Journal of Non-Linear Mechanics, vol. 35, no. 1, pp. 37-43, 2000. 


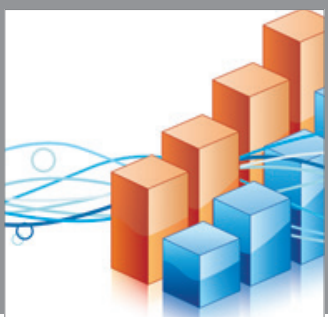

Advances in

Operations Research

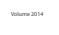

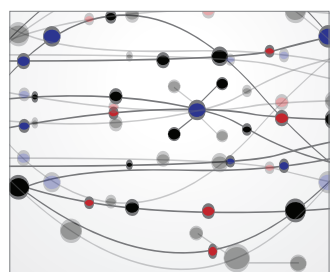

\section{The Scientific} World Journal
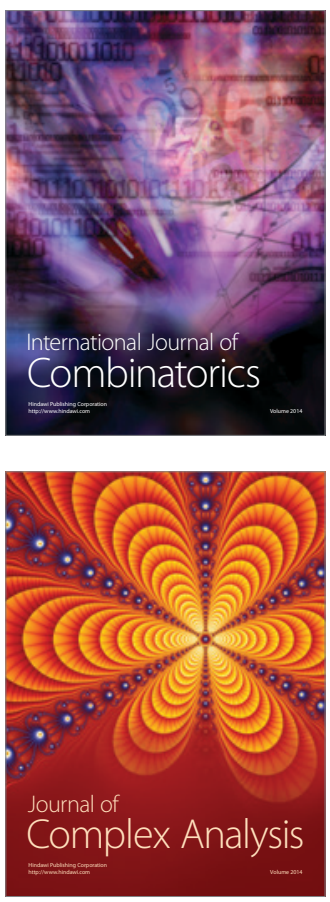

International Journal of

Mathematics and

Mathematical

Sciences
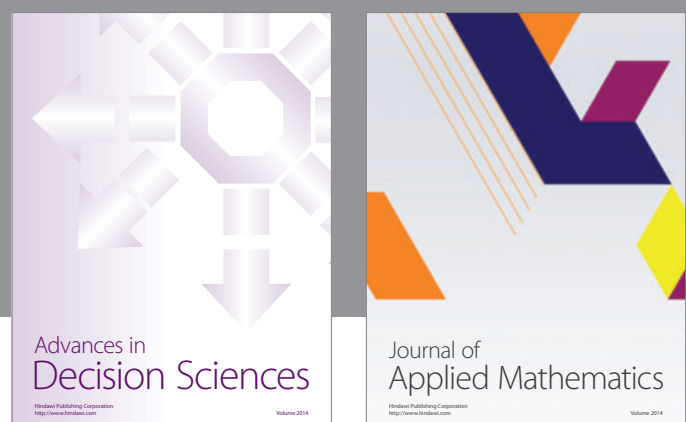

Journal of

Applied Mathematics
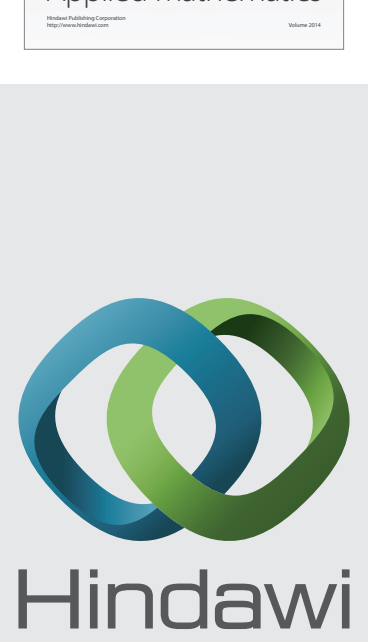

Submit your manuscripts at http://www.hindawi.com
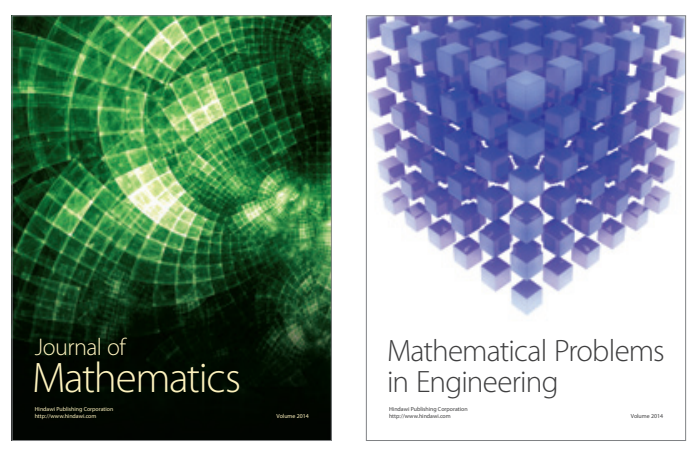

Mathematical Problems in Engineering
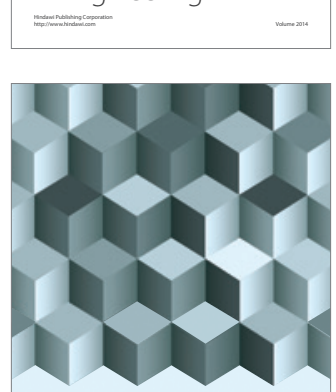

Journal of

Function Spaces
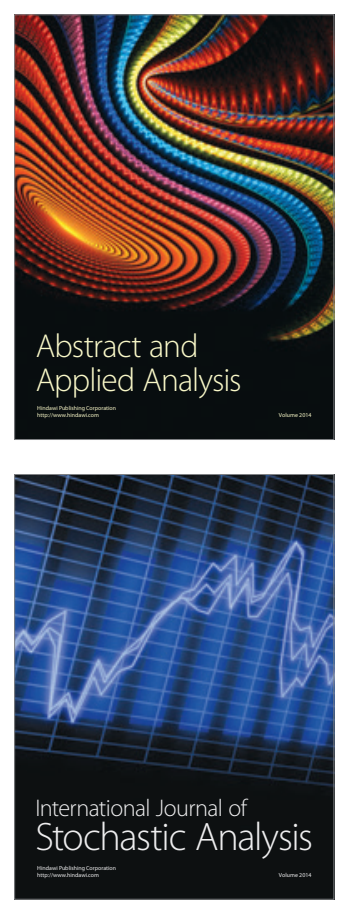

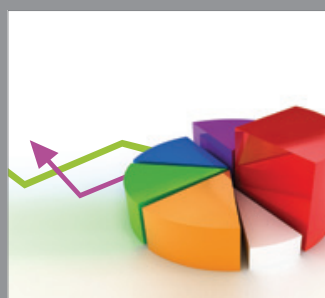

ournal of

Probability and Statistics

Promensencen
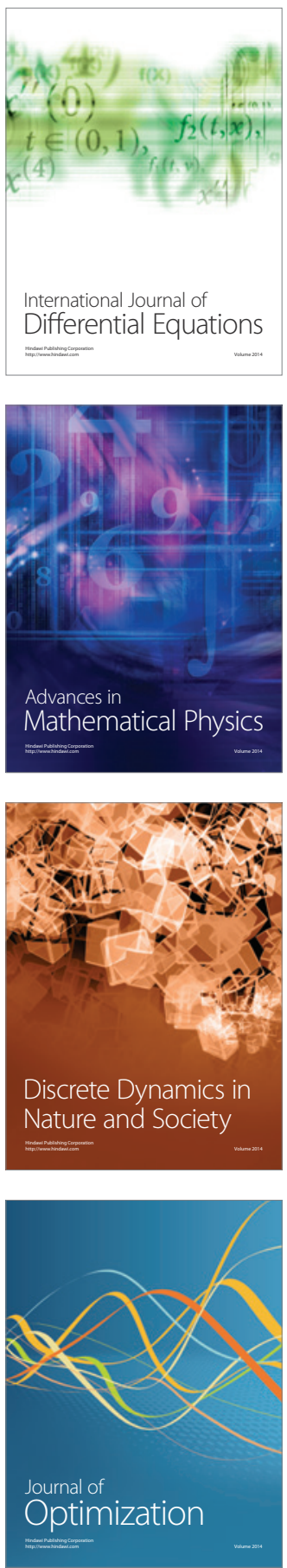